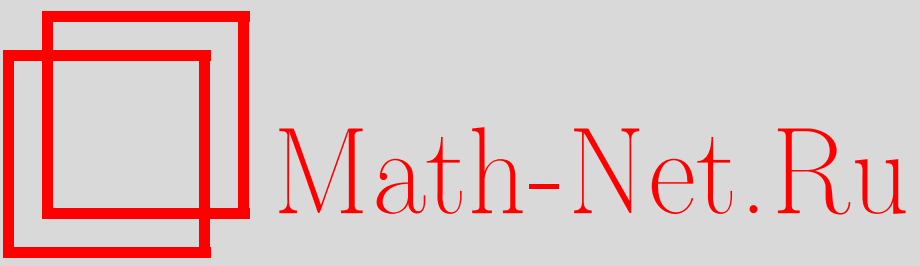

М. Гиат, С. Камуш, А. Хеллаф, В. Мерчела, Об одной системе интегральных уравнений Вольтерра со слабо сингулярным ядром, Итоги науки и техн. Сер. Соврем. мат. и ее прил. Темат. обз., 2021, том 193, 33-44

DOI: https://doi.org/10.36535/0233-6723-2021-193-33-44

Использование Общероссийского математического портала Math-Net.Ru подразумевает, что вы прочитали и согласны с пользовательским соглашением

http: //www. mathnet.ru/rus/agreement

Параметры загрузки:

IP : 34.239 .49 .27

26 апреля 2023 г., $16: 23: 45$ 


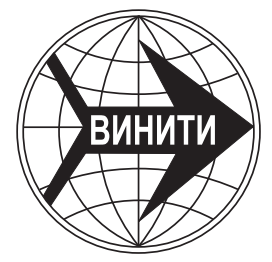

ИТОГИ НАУКИ И ТЕХНИКИ.

Современная математика и ее приложения.

Тематические обзоры.

Том 193 (2021). C. 33-44

DOI: 10.36535/0233-6723-2021-193-33-44

УДК 517.968

\title{
ОБ ОДНОЙ СИСТЕМЕ ИНТЕГРАЛЬНЫХ УРАВНЕНИЙ ВОЛЬТЕРРА СО СЛАБО СИНГУЛЯРНЫМ ЯДРОМ
}

\author{
(c) 2021 г. М. ГИАТ, С. КАМУШ, А. ХЕЛЛАФ, В. МЕРЧЕЛА
}

\begin{abstract}
АннотАция. В работе исследуется существование и единственность решений системы интегральных уравнений Вольтерра со слабо сингулярным ядром. Аппроксимация решения производится при помощи метода интегрирования произведения. Точность и эффективность метода проиллюстрированы на некоторых численных примерах.
\end{abstract}

Ключевые слова: нелинейное интегральное уравнение Вольтерра, интегро-дифференциальное уравнение, неподвижная точка, метод интегрирования произведения.

\section{ON A SYSTEM OF VOLTERRA INTEGRAL EQUATIONS WITH A WEAKLY SINGULAR KERNEL}

\author{
(c) 2021 M. GHIAT, S. KAMOUCHE, A. KHELLAF, W. MERCHELA
}

\begin{abstract}
In this paper, we examine the existence and uniqueness of solutions for a system of Volterra integral equations with a weakly singular kernel. We approximate the solution of this system using the product integration method. The accuracy and efficiency of this method are illustrated in some numerical examples.
\end{abstract}

Keywords and phrases: nonlinear Volterra integral equation, integro-differential equation, fixed point, product integration method.

AMS Subject Classification: 45D05, 65D07, 65R20.

1. Введение. Многие физические процессы и математические модели описываются нелинейными интегральными уравнениями Вольтерры (см. [1,2,6-8]). Уравнения этого типа привлекают внимание многих исследователей. В данной работе рассматриваются нелинейные интегральные уравнения Вольтерры со слабо-сверточными сингулярными ядрами, которые определяются следующим образом: найти функции $x(t)$ и $y(t)$, определенные для всех $t \in[a, b]$ и удовлетворяющие условиям

$$
\left\{\begin{array}{l}
x(t)=\int_{a}^{t} p_{1}(t-s) K_{1}(t, s, x(s), y(s)) d s+f_{1}(t), \\
y(t)=\int_{a}^{t} p_{2}(t-s) K_{2}(t, s, x(s), y(s)) d s+f_{2}(t),
\end{array}\right.
$$

где $f_{1}, f_{2}, p_{1}, p_{2}, K_{1}, K_{2}$ - заданные функции. Отметим, что многие интегральные уравнения типа Вольтерры, возникающие в прикладных задачах, имеют сверточные ядра (см. [3]). 
Отметим, что интегро-дифференциальные нелинейные уравнения Вольтерры вида

$$
u(t)=f(t)+\int_{0}^{t} k\left(s, u(s), u^{\prime}(s)\right) d s, \quad t \in[0,1],
$$

рассматривались в $[5,9]$, а уравнения вида

$$
u(t)=f(t)+\int_{0}^{t} p(t-s) k\left(s, u(s), u^{\prime}(s)\right) d s, \quad t \in[0,1]
$$

- в [4]; здесь $f, k$ и $p$-заданные функция, $u$-неизвестная. В [4] при исследовании условий на функцию $p(\cdot)$ было установлено, что уравнение (1) эквивалентно системе нелинейных интегральных уравнений вида

$$
\left\{\begin{array}{l}
u(t)=\int_{a}^{t} p(t-s) k\left(t, s, u(s), u^{\prime}(s)\right) d s+f(t) \\
u^{\prime}(t)=\int_{a}^{t} p^{\prime}(t-s) k\left(s, u(s), u^{\prime}(s)\right) d s+\int_{a}^{t} p(t-s) \frac{\partial k}{\partial t}\left(t, s, u(s), u^{\prime}(s)\right) d s+f^{\prime}(t),
\end{array}\right.
$$

для всех $t \in[a, b]$, причем сингулярность определяется только функиией $p^{\prime}(\cdot)$.

$\mathrm{B}$ данной работе мы получим условия на функции $p_{1}(\cdot)$ и $p_{2}(\cdot)$ в системе $(\mathrm{P} 1)$, обобщающие аналитическое и численное исследование системы (Р2), и, как следствие, уравнение (1). Отметим, что сингулярность задается для $p_{1}(\cdot)$ и $p_{2}(\cdot)$.

Далее система (P1) исследуется аналитически; существование решения доказано при помощи теоремы Шаудера о неподвижной точке, а единственность - при помощи методов, предложенных в [4]. Далее приводятся результаты численных экспериментов, проведенных при помощи метода интегрирования произведения (см. [6]).

Отметим, что использование классических методов (например, метод Нистрома) для поиска приближенного решения системы (P1) в рассматриваемом случае невозможно, в то время как применение метода интегрирования произведения позволяет обойти сингулярности функций $p_{1}(\cdot)$ и $p_{2}(\cdot)$. Кроме того, этот метод позволяет обобщить рассмотрение на случай системы типа (P1) с $n$ неизвестными функциями, $n \geqslant 2$.

Пусть $p_{1}, p_{2}, f_{1}, f_{2}, K_{1}, K_{2}$ - вещественные функции, удовлетворяющие следующим условиям:

$$
\begin{array}{rlrl}
p_{i}:[a-b, b-a] \rightarrow \mathbb{R}, & t \mapsto p_{i}(t), \\
f_{i}:[a, b] \rightarrow \mathbb{R}, & t \mapsto f_{i}(t), \\
K_{i}:[a, b]^{2} \times \mathbb{R}^{2} \rightarrow \mathbb{R}, & & (t, s, x, y) \mapsto K_{i}(t, s, x, y),
\end{array}
$$

где $i=1,2$. Также предположим, что

$$
\begin{gathered}
f_{1}, f_{2} \in \mathcal{C}([a, b]), \quad K_{1}, K_{2} \in \mathcal{C}\left([a, b]^{2} \times \mathbb{R}^{2}\right), \\
\exists M_{1}, M_{2}>0 \forall t, s \in[a, b] \forall x, y \in \mathbb{R}: \quad\left|K_{1}(t, s, x, y)\right| \leqslant M_{1}, \quad\left|K_{2}(t, s, x, y)\right| \leqslant M_{2} ; \\
p_{1}, p_{2} \in L^{1}(a-b, b-a), \quad \lim _{\tau \rightarrow 0} p_{1}(\tau) d s=+\infty, \quad \lim _{\tau \rightarrow 0} p_{2}(\tau) d s=+\infty, \\
\forall t \in[a, b]: \lim _{\delta \rightarrow 0^{+}} \int_{t}^{t+\delta}\left|p_{1}(t+\delta-s)\right| d s=0, \quad \lim _{\delta \rightarrow 0^{+}} \int_{t}^{t+\delta}\left|p_{2}(t+\delta-s)\right| d s=0 .
\end{gathered}
$$


Условие (Н2) объясняет сингулярность в системе (Р1): эта сингулярность является слабой, поскольку для всех $t \in[a, b]$

$$
\int_{a}^{t}\left|p_{i}(t-s)\right| d s \leqslant \int_{0}^{t-a}\left|p_{i}(\tau)\right| d \tau \leqslant \int_{a-b}^{b-a}\left|p_{i}(\tau)\right| d \tau<\infty, \quad i=1,2 .
$$

Наша задача - найти условия, обеспечивающие существование и единственность решения системы (P1) и разработать численный алгоритм поиска этого решения. Отметим, что предположение (H1) аналогично условиям, наложенным в $[4,5]$. В дальнейшем будем обозначать через $\|\cdot\|_{\infty}$ и $\|\cdot\|_{1}$ нормы банаховых пространств $\mathcal{C}([a, b])$ и $L^{1}(a-b, b-a)$, соответственно.

2. Аналитическое исследование. Докажем, что система (P1) имеет единственное решение в $\mathcal{C}([a, b]) \times \mathcal{C}([a, b])$. Введем некоторые определения.

Модуль непрерывности функции $x$ в $\mathcal{C}([a, b])$ :

$$
\omega_{0}(x, h)=\sup _{|t-\sigma| \leqslant h}|x(t)-x(\sigma)| .
$$

Колебание функции $q$ в $L^{1}(a-b, b-a)$ :

$$
\omega_{1}(q, h)=\sup _{|t-\sigma| \leqslant h} \int_{a-b}^{b-a}|q(t-s)-q(\sigma-s)| d s .
$$

Модуль переноса функции $q$ in $L^{1}(a-b, b-a)$ :

$$
\tau(q, h)=\int_{t}^{t+h}|q(t+h-s)| d s .
$$

Докажем некоторые вспомогательные факты.

Лемма 1. $Е \operatorname{cлu} x \in \mathcal{C}([a, b])$, mo $\lim _{h \rightarrow 0} \omega_{0}(x, h)=0$.

Доказательство. Это утверждение вытекает из равномерной непрерывности функции $x$ на компакте $[a, b]$.

Лемма 2. Если $q \in L^{1}(a-b, b-a), m o \lim _{h \rightarrow 0} \omega_{1}(q, h)=0$.

Доказательство. Используем тот факт, что $\mathcal{C}([a-b, b-a])$ плотно в $L^{1}(a-b, b-a)$ и затем применим лемму 1.

Из (Н2) при $i=1,2$ имеем

$$
\lim _{h \rightarrow 0} \tau\left(p_{i}, h\right)=0 .
$$

Теорема 1. При выполнении условий (H1) и (H2) система (P1) имеем решение $(x, y) \in$ $\mathcal{C}([a, b]) \times \mathcal{C}([a, b])$.

Доказательство. Для $f_{1}, f_{2} \in \mathcal{C}([a, b])$ рассмотрим множество

$$
\mathcal{D}=\left\{(x, y) \in \mathcal{C}([a, b]) \times \mathcal{C}([a, b]): \quad \begin{array}{l}
x(a)=f_{1}(a),\left\|x-f_{1}\right\|_{\infty} \leqslant M_{1}\left\|p_{1}\right\|_{1} \\
y(a)=f_{2}(a),\left\|y-f_{2}\right\|_{\infty} \leqslant M_{2}\left\|p_{2}\right\|_{1}
\end{array}\right\} .
$$

Для всех $(x, y) \in \mathcal{D}$ определим функционал

$$
\Phi(x, y)(t)=\left(\begin{array}{l}
\Phi_{f_{1}}(x, y)(t) \\
\Phi_{f_{2}}(x, y)(t)
\end{array}\right)=\left(\begin{array}{c}
f_{1}(t)+\int_{a}^{t} p_{1}(t-s) K_{1}(t, s, x(s), y(s)) d s \\
f_{2}(t)+\int_{a}^{t} p_{2}(t-s) K_{2}(t, s, x(s), y(s)) d s
\end{array}\right)
$$


Ясно, что множество $\mathcal{D}$ замкнуто и выпукло в $\mathcal{C}([a, b]) \times \mathcal{C}([a, b])$. Далее, для всех $(x, y) \in \mathcal{D}$, $t \in[a, b], i=1,2$ имеем

$$
\Phi_{f_{i}}(x, y)(a)=f_{i}(a)
$$

$$
\begin{aligned}
\left|\Phi_{f_{i}}(x, y)(t)-f_{i}(t)\right|= & \left|\int_{a}^{t} p_{i}(t-s) K_{i}(t, s, x(s), y(s)) d s\right| \leqslant \\
& \leqslant \int_{a}^{t}\left|p_{i}(t-s)\right|\left|K_{i}(t, s, x(s), y(s))\right| d s \leqslant M_{i} \int_{a}^{t}\left|p_{i}(t-s)\right| d s \leqslant M_{i}\left\|p_{i}\right\|_{1} .
\end{aligned}
$$

Отсбда заключаем, что $\Phi(\mathcal{D}) \subset \mathcal{D}$. Пусть $\left(x_{n}, y_{n}\right)_{n \in \mathbb{N}}$ - последовательность в $\mathcal{D}$, сходящаяся к $(x, y) \in \mathcal{D}$. для всех $t \in[a, b]$ и $i=1,2$ имеем

$$
\begin{aligned}
\lim _{n \rightarrow+\infty} \Phi_{f_{i}}\left(x_{n}, y_{n}\right)(t)= & \lim _{n \rightarrow+\infty} \int_{a}^{t} p_{i}(t-s) K_{i}\left(t, s, x_{n}(s), y_{n}(s)\right) d s+f_{i}(t)= \\
& =\int_{a}^{t} \lim _{n \rightarrow+\infty} p_{i}(t-s) K_{i}\left(t, s, x_{n}(s), y_{n}(s)\right) d s+f_{i}(t)= \\
& =\int_{a}^{t} p_{i}(t-s) K_{i}\left(t, s, \lim _{n \rightarrow+\infty} x_{n}(s), \lim _{n \rightarrow+\infty} y_{n}(s)\right) d s+f_{i}(t)= \\
& =\int_{a}^{t} p_{i}(t-s) K_{i}(t, s, x(s), y(s)) d s+f_{i}(t)=\Phi_{f_{i}}(x, y)(t)
\end{aligned}
$$

откуда следует, что функционал $\Phi$ непрерывен на $\mathcal{D}$. С другой стороны, для $t \in[a, b]$ и $(x, y) \in \mathcal{D}$ имеем

$$
\begin{aligned}
\left|\Phi_{f_{i}}(x, y)(t+h)-\Phi_{f_{i}}(x, y)(t)\right| \leqslant & \\
\leqslant \int_{a}^{t} \mid p_{i}(t+h-s) & K_{i}(t+h, s, x(s), y(s))-p_{i}(t-s) K_{i}(t, s, x(s), y(s)) \mid d s+ \\
& +\int_{t}^{t+h}\left|p_{i}(t+h-s) K_{i}(t+h, s, x(s), y(s))\right| d s+\left|f_{i}(t+h)-f_{i}(t)\right| .
\end{aligned}
$$

Таким образом,

$$
\begin{aligned}
\int_{a}^{t} \mid p_{i}(t+h-s) & K_{i}(t+h, s, x(s), y(s))- \\
& p_{i}(t-s) K_{i}(t, s, x(s), y(s)) \mid d s \leqslant \\
\leqslant & \int_{a}^{t}\left|p_{i}(t-s)\right|\left|K_{i}(t+h, s, x(s), y(s))-K_{i}(t, s, x(s), y(s))\right| d s+ \\
& +\int_{a}^{t}\left|p_{i}(t+h-s)-p_{i}(t-s)\right| \cdot\left|K_{i}(t+h, s, x(s), y(s))\right| d s .
\end{aligned}
$$

Используя условие $(\mathrm{H} 1)$, можем найти такую точку $(\tilde{s}, \tilde{x}, \tilde{y}) \in[a, b] \times \mathbb{R}^{2}$, что

$$
\left|K_{i}(t+h, s, x(s), y(s))-K_{i}(t, s, x(s), y(s))\right| \leqslant\left|K_{i}(t+h, \tilde{s}, \tilde{x}, \tilde{y})-K_{i}(t, \tilde{s}, \tilde{x}, \tilde{y})\right|
$$


для всех $t \in[a, b]$. Если ввести обозначение $\tilde{K}_{i}(t)=K_{i}(t, \tilde{s}, \tilde{x}, \tilde{y})$ для $t \in[a, b]$, то

$$
\left|K_{i}(t+h, s, x(s), y(s))-K_{i}(t, s, x(s), y(s))\right| \leqslant \omega_{0}\left(\tilde{K}_{i}, h\right) .
$$

Кроме того, нетрудно видеть, что

$$
\begin{aligned}
\int_{a}^{t} \mid p_{i}(t+h-s) & -p_{i}(t-s)|\cdot| K_{i}(t+h, s, x(s), y(s))\left|d s \leqslant M_{i} \int_{a}^{t}\right| p_{i}(t+h-s)-p_{i}(t-s) \mid d s \leqslant \\
& \leqslant M_{i} \int_{0}^{t-a}\left|p_{i}(\sigma+h)-p_{i}(\sigma)\right| d \sigma \leqslant M_{i} \int_{a-b}^{b-a}\left|p_{i}(\sigma+h)-p_{i}(\sigma)\right| d \sigma \leqslant M_{i} \omega_{1}\left(p_{i}, h\right) .
\end{aligned}
$$

Из (12) и (13) заключаем

$$
\begin{aligned}
\int_{a}^{t}\left|p_{i}(t+h-s) K_{i}(t+h, s, x(s), y(s))-p_{i}(t-s) K_{i}(t, s, x(s), y(s))\right| d s & \\
& \leqslant \omega_{0}\left(\tilde{K}_{i}, h\right)\left\|p_{i}\right\|_{1}+M_{i} \omega_{1}\left(p_{i}, h\right) .
\end{aligned}
$$

Итак,

$$
\left|\Phi_{f_{i}}(x, y)(t+h)-\Phi_{f_{i}}(x, y)(t)\right| \leqslant \omega_{0}\left(\tilde{K}_{i}, h\right)\left\|p_{i}\right\|_{1}+M_{i} \omega_{1}\left(p_{i}, h\right)+M_{i} \tau\left(p_{i}, h\right)+\omega_{0}\left(f_{i}, h\right) .
$$

Согласно леммам 1 и 2 , принимая во внимание последнее равенство, заключаем, что функционал $\Phi(x, y)(\cdot)$ равномерно непрерывен на $\mathcal{D}$. Окончательно, используя теорему Шаудера, получаем, что $\Phi_{f}$ имеем неподвижную точку в $\mathcal{D}$, а Это и означает, что система $(\mathrm{P} 1)$ имеет решение в $\mathcal{C}([a, b]) \times \mathcal{C}([a, b])$.

Отметим, что в [4] было наложено больше условий и использовалась теорема Банаха о неподвижной точке. Таким образом, теорема 1 с условием (Н1) является более общей.

Для доказательства единственности наложим следующие условия:

$$
\exists L_{i}, \tilde{L}_{i} \in \mathbb{R}_{+}^{*} \forall t, s \in[a, b], \forall x, y, \tilde{x}, \tilde{y} \in \mathbb{R}:
$$

$$
\left|K_{i}(t, s, x, y)-K_{i}(t, s, \tilde{x}, \tilde{y})\right| \leqslant L_{i}|x-\tilde{x}|+\tilde{L}_{i}|y-\tilde{y}|, \quad i=1,2 .
$$

Пусть $\left\{T_{j}\right\}_{0 \leqslant j \leqslant k}$ - такое разбиение

$$
a=T_{0} \leqslant T_{1} \leqslant \ldots \leqslant T_{k}=b
$$

интервала $[a, b]$, что существует $\rho>0$ для которого при всех $t, s \in\left[T_{j}, T_{j+1}\right]$ имеем

$$
\max \left(L_{i}, \tilde{L}_{i}\right) \int_{T_{j}}^{\min \left(t, T_{j+1}\right)}\left|p_{i}(t-s)\right| d s \leqslant \rho<\frac{1}{2} .
$$

Отметим, что условие (Н2') сожно вывести из условия (Н2). Сначала докажем единственность решения системы $(\mathrm{P} 1)$ на интервале $\left[T_{0}, T_{1}\right]$, затем продолжим это решение на $\left[T_{1}, T_{2}\right]$ и т. д.

Теорема 2. При выполнении условий (Н3) решение системъ $(\mathrm{P} 1)$ единственно на $\mathcal{C}\left(\left[T_{0}, T_{1}\right]\right) \times$ $\mathcal{C}\left(\left[T_{0}, T_{1}\right]\right)$ 
Доказательство. Предположим, что существуют два решения $(x, y)$ и $(\tilde{x}, \tilde{y})$ системы $(\mathrm{P} 1)$. Тогда для $t \in\left[T_{0}, T_{1}\right]$ имеем

$$
\begin{gathered}
|x(t)-\tilde{x}(t)| \leqslant \int_{a}^{t}\left|p_{1}(t-s)\right|\left|K_{1}(t, s, x(s), y(s))-K_{1}(t, s, \tilde{x}(s), \tilde{y}(s))\right| d s \leqslant \\
\leqslant \int_{a}^{t}\left|p_{1}(t-s)\right|\left[L_{1}|x(s)-\tilde{x}(s)|+\tilde{L_{1}}|y(s)-\tilde{y}(s)|\right] d s \leqslant \\
\leqslant\left[\max _{T_{0} \leqslant t \leqslant T_{1}}\left|x_{1}(t)-x_{2}(t)\right|+\max _{T_{0} \leqslant t \leqslant T_{1}}\left|y_{1}(t)-y_{2}(t)\right|\right] \max \left(L_{1}, \tilde{L_{1}}\right) \int_{a}^{t}\left|p_{1}(t-s)\right| d s \leqslant \\
\quad \leqslant \rho\left[\max _{T_{0} \leqslant t \leqslant T_{1}}|x(t)-\tilde{x}(t)|+\max _{T_{0} \leqslant t \leqslant T_{1}}|y(t)-\tilde{y}(t)|\right] .
\end{gathered}
$$

Аналогично,

$$
\begin{aligned}
|y(t)-\tilde{y}(t)| \leqslant \int_{a}^{t}\left|p_{2}(t-s)\right| \mid K_{2}(t, s, x(s), y(s))- & K_{2}(t, s, \tilde{x}(s), \tilde{y}(s)) \mid d s \leqslant \\
& \leqslant \rho\left[\max _{T_{0} \leqslant t \leqslant T_{1}}|x(t)-\tilde{x}(t)|+\max _{T_{0} \leqslant t \leqslant T_{1}}|y(t)-\tilde{y}(t)|\right] .
\end{aligned}
$$

Таким образом,

$$
\max _{T_{0} \leqslant t \leqslant T_{1}}|x(t)-\tilde{x}(t)|+\max _{T_{0} \leqslant t \leqslant T_{1}}|y(t)-\tilde{y}(t)| \leqslant 2 \rho\left[\max _{T_{0} \leqslant t \leqslant T_{1}}|x(t)-\tilde{x}(t)|+\max _{T_{0} \leqslant t \leqslant T_{1}}|y(t)-\tilde{y}(t)|\right] .
$$

Поскольку $\rho<1 / 2$, заключаем, что $(1-2 \rho)>0$; следовательно, $x(t)=\tilde{x}(t)$ и $y(t)=\tilde{y}(t)$ при $t \in\left[T_{0}, T_{1}\right]$.

Продолжим найденное решение на весь интервал $[a, b]$.

Теорема 3. Система $(\mathrm{P} 1)$ имеем единственное решение $(x, y) \in \mathcal{C}([a, b]) \times \mathcal{C}([a, b])$.

Доказательство. Обозначим через $\left(x_{0}, y_{0}\right)$ решение системы $(\mathrm{P} 1)$ на $\left[T_{0}, T_{1}\right]$, а через $\left(x_{1}, y_{1}\right)-$ решение системы (P1) на $t \in\left[T_{1}, T_{2}\right]$ :

$$
\left\{\begin{array}{l}
x_{1}(t)=f_{1}(t)+\int_{T_{0}}^{T_{1}} p_{1}(t-s) K_{1}\left(t, s, x_{0}(s), y_{0}(s)\right) d s+\int_{T_{1}}^{t} p_{1}(t-s) K_{1}\left(t, s, x_{1}(s), y_{1}(s)\right) d s \\
y_{1}(t)=f_{2}(t)+\int_{T_{0}}^{T_{1}} p_{2}(t-s) K_{2}\left(t, s, x_{0}(s), y_{0}(s)\right) d s+\int_{T_{1}}^{t} p_{2}(t-s) K_{2}\left(t, s, x_{1}(s), y_{1}(s)\right) d s .
\end{array}\right.
$$

Доказательство существования и единственности решения $\left(x_{1}, y_{1}\right)$ на $\left[T_{1}, T_{2}\right]$ проводится так же, как и выше. Определим теперь решение системы (P1) на $\left[T_{0}, T_{2}\right]$ следующим образом:

$$
x(t)=\left\{\begin{array}{ll}
x_{0}(t), \quad t \in\left[T_{0}, T_{1}\right], \\
x_{1}(t), \quad t \in\left[T_{1}, T_{2}\right],
\end{array} \quad y(t)= \begin{cases}y_{0}(t), & t \in\left[T_{0}, T_{1}\right], \\
y_{1}(t), & t \in\left[T_{1}, T_{2}\right] .\end{cases}\right.
$$

Ясно, что $(x, y) \in \mathcal{C}\left(\left[T_{0}, T_{2}\right]\right) \times \mathcal{C}\left(\left[T_{0}, T_{2}\right]\right)$, откуда следует, что система (Р1) имеет единственное решение на $\left[T_{0}, T_{2}\right]$. Продолжая аналогичным образом, можно построить решение на $[a, b]$. 
3. Численный эксперимент. Система (Р1) содержит ядра, состоящие из двух слагаемых: регулярных $K_{i}$ и слабо сингулярных $p_{i}, i=1,2$. Для устранения сингулярностей применим метод интегрирования произведения. Построим приближения регулярных частей $K_{1}, K_{2}$.

Пусть $\left\{t_{j}\right\}_{0 \leqslant j \leqslant n}-$ разбиение интервала, где $t_{j}=a+j h$ и $h=(b-a) / n$. Введем следующие обозначения:

$$
\begin{aligned}
& P_{n, 1}\left[K_{1}\right](t, s, x(s), y(s))=\frac{s-t_{j}}{h} K_{1}\left(t, t_{j+1}, x\left(t_{j+1}\right), y\left(t_{j+1}\right)\right)+\frac{t_{j+1}-s}{h} K_{1}\left(t, t_{j}, x\left(t_{j}\right), y\left(t_{j}\right)\right), \\
& P_{n, 1}\left[K_{2}\right](t, s, x(s), y(s))=\frac{s-t_{j}}{h} K_{2}\left(t, t_{j+1}, x\left(t_{j+1}\right), y\left(t_{j+1}\right)\right)+\frac{t_{j+1}-s}{h} K_{2}\left(t, t_{j}, x\left(t_{j}\right), y\left(t_{j}\right)\right),
\end{aligned}
$$

где $t_{j} \leqslant s \leqslant t_{j+1}$; получим приближение следующих интегралов:

$$
\begin{aligned}
& \int_{a}^{t_{i}} p_{1}\left(t_{i}-s\right) K_{1}\left(t_{i}, s, x(s), y(s)\right) d s \simeq \gamma_{i, 1} K_{1}\left(t_{i}, t_{0}, x\left(t_{0}\right), y\left(t_{0}\right)\right)+\lambda_{i, i} K_{1}\left(t_{i}, t_{i}, x\left(t_{i}\right), y\left(t_{i}\right)\right)+ \\
&+\sum_{j=1}^{i-1}\left(\gamma_{i, j+1}+\lambda_{i, j}\right) K_{1}\left(t_{i}, t_{j}, x\left(t_{j}\right), y\left(t_{j}\right)\right), \\
& \int_{a}^{t_{i}} p_{2}\left(t_{i}-s\right) K_{2}\left(t_{i}, s, x(s), y(s)\right) d s \simeq \tilde{\gamma}_{i, 1} K_{2}\left(t_{i}, t_{0}, x\left(t_{0}\right), y\left(t_{0}\right)\right)+\tilde{\lambda}_{i, i} K_{2}\left(t_{i}, t_{n}, x\left(t_{i}\right), y\left(t_{i}\right)\right)+ \\
&+\sum_{j=1}^{i-1}\left(\tilde{\gamma}_{i, j+1}+\tilde{\lambda}_{i, j}\right) K_{2}\left(t_{i}, t_{j}, x\left(t_{j}\right), y\left(t_{j}\right)\right),
\end{aligned}
$$

где

$$
\begin{cases}\gamma_{i, j+1}=\frac{1}{h} \int_{t_{j}}^{t_{j+1}} p_{1}\left(t_{i}-s\right)\left(t_{j+1}-s\right) d s, & \lambda_{i, j+1}=\frac{1}{h} \int_{t_{j}}^{t_{j+1}} p_{1}\left(t_{i}-s\right)\left(s-t_{j}\right) d s, \\ \tilde{\gamma}_{i, j+1}=\frac{1}{h} \int_{t_{j}}^{t_{j+1}} p_{2}\left(t_{i}-s\right)\left(t_{j+1}-s\right) d s, & \tilde{\lambda}_{i, j+1}=\frac{1}{h} \int_{t_{j}}^{t_{j+1}} p_{2}\left(t_{i}-s\right)\left(s-t_{j}\right) d s\end{cases}
$$

для всех $0 \leqslant j \leqslant i-1$.

Приближенная система для (P1) получается следующим образом:

$$
\left\{\begin{array}{l}
X_{i}=f_{1}\left(t_{i}\right)+\gamma_{i, 1} K_{1}\left(t_{i}, t_{0}, X_{0}, Y_{0}\right)+\sum_{j=1}^{i-1}\left(\gamma_{i, j+1}+\lambda_{i, j}\right) K_{1}\left(t_{i}, t_{j}, X_{j}, Y_{j}\right)+\lambda_{i, i} K_{1}\left(t_{i}, t_{i}, X_{i}, Y_{i}\right), \\
Y_{i}=f_{2}\left(t_{i}\right)+\tilde{\gamma}_{i, 1} K_{2}\left(t_{i}, t_{0}, X_{0}, Y_{0}\right)+\sum_{j=1}^{i-1}\left(\tilde{\gamma}_{i, j+1}+\tilde{\lambda}_{i, j}\right) K_{2}\left(t_{i}, t_{j}, X_{j}, Y_{j}\right)+\tilde{\lambda}_{i, i} K_{2}\left(t_{i}, t_{i}, X_{i}, Y_{i}\right), \\
X_{0}=f_{1}(a), \quad Y_{0}=f_{2}(a),
\end{array}\right.
$$

для всех $0 \leqslant j \leqslant i-1$, где $X_{i}, Y_{i}$-приближения для $x\left(t_{i}\right)$ и $y\left(t_{i}\right)$.

Теорема 4. При достаточно мальх $h$ система (Г) имеет единственное решение.

Доказательство. Введем обозначение

Для всех $1 \leqslant i \leqslant n$ положим

$$
\forall\left(\begin{array}{l}
X \\
Y
\end{array}\right) \in \mathbb{R}^{2}, \quad\left\|\left(\begin{array}{l}
X \\
Y
\end{array}\right)\right\|=|X|+|Y|
$$

$$
\Psi_{i}\left(\begin{array}{l}
X \\
Y
\end{array}\right):=\left(\begin{array}{l}
S_{1}+\lambda_{i, i} K_{1}\left(t_{i}, t_{i}, X, Y\right) \\
S_{2}+\tilde{\lambda}_{i, i} K_{2}\left(t_{i}, t_{i}, X, Y\right)
\end{array}\right)
$$


где

$$
\begin{aligned}
& S_{1}=f_{1}\left(t_{i}\right)+\gamma_{i, 1} K_{1}\left(t_{i}, t_{0}, X_{0}, Y_{0}\right)+\sum_{j=1}^{i-1}\left(\gamma_{i, j+1}+\lambda_{i, j}\right) K_{1}\left(t_{i}, t_{j}, X_{j}, Y_{j}\right), \\
& S_{2}=f_{2}\left(t_{i}\right)+\tilde{\gamma}_{i, 1} K_{2}\left(t_{i}, t_{0}, X_{0}, Y_{0}\right)+\sum_{j=1}^{i-1}\left(\tilde{\gamma}_{i, j+1}+\tilde{\lambda}_{i, j}\right) K_{2}\left(t_{i}, t_{j}, X_{j}, Y_{j}\right) .
\end{aligned}
$$

Предположив, что $X_{0}, X_{1}, \ldots, X_{i-1}$ и $Y_{0}, Y_{1}, \ldots, Y_{i-1}$ известны, получим систему неявных уравнений

$$
\left(\begin{array}{c}
X_{i} \\
Y_{i}
\end{array}\right)=\Psi_{i}\left(\begin{array}{c}
X_{i} \\
Y_{i}
\end{array}\right)
$$

Докажем, что $\Psi_{i}$ имеет единственную неподвижную точку,

$$
\begin{gathered}
\left\|\Psi_{i}\left(\begin{array}{l}
X \\
Y
\end{array}\right)-\Psi_{n}\left(\begin{array}{l}
X^{\prime} \\
Y^{\prime}
\end{array}\right)\right\|=\left\|\left(\begin{array}{l}
\eta_{1} \\
\eta_{2}
\end{array}\right)\right\|, \\
\eta_{1}=\lambda_{i, i}\left(K_{1}\left(t_{i}, t_{i}, X, Y\right)-K_{1}\left(t_{i}, t_{i}, X^{\prime}, Y^{\prime}\right)\right), \quad \eta_{2}=\tilde{\lambda}_{i, i}\left(K_{2}\left(t_{i}, t_{i}, X, Y\right)-K_{2}\left(t_{i}, t_{i}, X^{\prime}, Y^{\prime}\right)\right) .
\end{gathered}
$$

При достаточно малом $h$ имеем

$$
\begin{aligned}
& \left|\eta_{1}\right| \leqslant \lambda_{i, i}\left(L_{1}\left|X-X^{\prime}\right|+\tilde{L}_{1}\left|Y-Y^{\prime}\right|\right) \leqslant \max \left(L_{1}, \tilde{L}_{1}\right) \lambda_{i, i}\left(\left|X-X^{\prime}\right|+\left|Y-Y^{\prime}\right|\right), \\
& \left|\eta_{2}\right| \leqslant \tilde{\lambda}_{i, i}\left(L_{2}\left|X-X^{\prime}\right|+\tilde{L_{2}}\left|Y-Y^{\prime}\right|\right) \leqslant \max \left(L_{2}, \tilde{L_{2}}\right) \tilde{\lambda}_{i, i}\left(\left|X-X^{\prime}\right|+\left|Y-Y^{\prime}\right|\right) .
\end{aligned}
$$

Таким образом,

$$
\left\|\Psi_{i}\left(\begin{array}{l}
X \\
Y
\end{array}\right)-\Psi_{i}\left(\begin{array}{l}
X^{\prime} \\
Y^{\prime}
\end{array}\right)\right\| \leqslant \max \left(L_{1}, \tilde{L}_{1}, L_{2}, \tilde{L_{2}}\right) \max \left(\lambda_{i, i}, \tilde{\lambda}_{i, i}\right)\left\|\left(\begin{array}{l}
X \\
Y
\end{array}\right)-\left(\begin{array}{l}
X^{\prime} \\
Y^{\prime}
\end{array}\right)\right\| .
$$

Следовательно, $\lim _{h \rightarrow 0} \max \left(\lambda_{i, i}, \tilde{\lambda}_{i, i}\right)=0$. Согласно теореме Банаха о неподвижной точке $\Psi_{i}$ является сжимающим отображением при достаточно малом $h$. Таким образом, система $(\Gamma)$ имеет единственное решение $\left\{\left(X_{i}, Y_{i}\right)\right\}_{1 \leqslant i \leqslant n}$.

Докажем сходимость этого метода. Сначала установим согласованность. Ошибка согласованности - это величина

где

$$
\Delta\left(h, t_{i}\right)=\left(\begin{array}{l}
\delta\left(h, t_{i}\right) \\
\hat{\delta}\left(h, t_{i}\right)
\end{array}\right)
$$

$$
\begin{aligned}
\delta\left(h, t_{i}\right) & =\int_{a}^{t_{i}} p_{1}\left(t_{i}-s\right) K_{1}\left(t_{i}, s, x(s), y(s)\right) d s-\sum_{j=0}^{i} \mu_{i j} K_{1}\left(t_{i}, t_{j}, x\left(t_{j}\right), y\left(t_{j}\right)\right), \\
\hat{\delta}\left(h, t_{i}\right) & =\int_{t_{0}}^{t_{i}} p_{2}\left(t_{i}-s\right) K_{2}\left(t_{i}, s, x(s), y(s)\right) d s-\sum_{j=0}^{i} \hat{\mu}_{i j} K_{2}\left(t_{i}, t_{j}, x\left(t_{j}\right), y\left(t_{j}\right)\right),
\end{aligned}
$$

причем

$$
\mu_{i j}=\left\{\begin{array}{ll}
\gamma_{i, 1} & \text { при } j=0, \\
\gamma_{i, j+1}+\lambda_{i, j} & \text { при } 1 \leqslant j \leqslant i-1, \\
\lambda_{i, i} & \text { при } j=i ;
\end{array} \quad \hat{\mu}_{i j}= \begin{cases}\tilde{\gamma}_{i, 1} & \text { при } j=0, \\
\tilde{\gamma}_{i, j+1}+\tilde{\lambda}_{i, j} & \text { при } 1 \leqslant j \leqslant i-1, \\
\tilde{\lambda}_{i, i} & \text { при } j=i .\end{cases}\right.
$$

Предложение 1. Если $\Delta\left(h, t_{i}\right)$ - непрерывная функиия, то для всех $t \in[a, b]$ u $i=1,2, \ldots, n$ имеем

$$
\begin{aligned}
\max _{1 \leqslant i \leqslant n}\left|\Delta\left(h, t_{i}\right)\right| \leqslant \max _{\substack{a \leqslant t \leqslant b ; \\
x, y \in \mathbb{R}}} \omega_{0}\left(h, K_{1}(t, \cdot, x, y)\right)\left\|p_{1}\right\|_{1} & +\max _{\substack{a \leqslant t \leqslant b ; \\
x, y \in \mathbb{R}}} \omega_{0}\left(h, K_{2}(t, \cdot, x, y)\right)\left\|p_{2}\right\|_{1}+ \\
& +2 \rho\left(\omega_{0}\left(h, f_{1}\right)+\omega_{0}\left(h, f_{2}\right)+\omega\left(h, H_{1}\right)+\omega\left(h, H_{2}\right)\right),
\end{aligned}
$$


где

$$
H_{1}(t)=\int_{a}^{t} p_{1}(t-s) K_{1}(t, s, x(s), y(s)) d s, \quad H_{2}(t)=\int_{a}^{t} p_{2}(t-s) K_{2}(t, s, x(s), y(s)) d s .
$$

Доказательство. Известно, что

$$
\begin{aligned}
\left|\delta\left(h, t_{i}\right)\right| \leqslant \max _{|\tau-\theta|<h} & \left|K_{1}\left(t_{i}, \tau, x(\tau), y(\tau)\right)-K_{1}\left(t_{i}, \tau, x(\theta), y(\theta)\right)\right|+ \\
& +\left|K_{1}\left(t_{i}, \tau, x(\theta), y(\theta)\right)-K_{1}\left(t_{i}, \theta, x(\theta), y(\theta)\right)\right| \int_{a}^{t_{n}}\left|p_{1}\left(t_{i}-s\right)\right| d s \leqslant \\
& \leqslant \max _{\substack{a \leqslant t \leqslant b ; \\
x, y \in \mathbb{R}}}\left|K_{1}(t, \tau, x, y)-K_{1}(t, \theta, x, y)\right| \int_{a}^{t_{i}}\left|p_{1}\left(t_{i}-s\right)\right| d s+\rho\left(\omega_{0}(h, x)+\omega_{0}(h, y)\right),
\end{aligned}
$$

но

$$
x(\theta)-x(\tau)=f_{1}(\theta)-f_{1}(\tau)+\int_{a}^{\theta} p_{1}(\theta-s) K_{1}(\theta, s, x(s), y(s)) d s-\int_{a}^{\tau} p_{1}(\tau-s) K_{1}(\tau, s, x(s), y(s)) d s .
$$

Тогда

$$
\begin{gathered}
\max _{|\tau-\theta|<h}|x(\theta)-x(\tau)| \leqslant \max _{|\tau-\theta|<h}\left|f_{1}(\theta)-f_{1}(\tau)\right|+\max _{|\tau-\theta|<h}\left|H_{1}(\theta)-H_{1}(\tau)\right|, \\
\omega_{0}(h, x) \leqslant \omega_{0}\left(h, f_{1}\right)+\omega_{0}\left(h, H_{1}\right)
\end{gathered}
$$

получаем

$$
\omega_{0}(h, y) \leqslant \omega_{0}\left(h, f_{2}\right)+\omega_{0}\left(h, H_{2}\right)
$$

Таким образом,

$$
\left|\delta\left(h, t_{i}\right)\right| \leqslant \rho\left(\omega_{0}\left(h, f_{1}\right)+\omega_{0}\left(h, f_{2}\right)+\omega_{0}\left(h, H_{1}\right)+\omega_{0}\left(h, H_{2}\right)\right)+\max _{a \leqslant t \leqslant b ; x, y \in \mathbb{R}} \omega_{0}\left(h, K_{1}(t, \cdot, x, y)\right)\left\|p_{1}\right\|_{1} .
$$

Повторяя аналогичные шаги для $\hat{\delta}\left(h, t_{i}\right)$, получим

$$
\left|\hat{\delta}\left(h, t_{i}\right)\right| \leqslant \rho\left(\omega_{0}\left(h, f_{1}\right)+\omega_{0}\left(h, f_{2}\right)+\omega_{0}\left(h, H_{1}\right)+\omega_{0}\left(h, H_{2}\right)\right)+\max _{a \leqslant t \leqslant b ; x, y \in \mathbb{R}} \omega_{0}\left(h, K_{2}(t, \cdot, x, y)\right)\left\|p_{2}\right\|_{1},
$$

откуда следует, что

$$
\begin{aligned}
\max _{1 \leqslant i \leqslant n}\left|\Delta\left(h, t_{i}\right)\right| \leqslant \max _{\substack{a \leqslant t b ; \\
x, y \in \mathbb{R}}} \omega_{0}\left(h, K_{1}(t, \cdot, x, y)\right)\left\|p_{1}\right\|_{1}+\max _{\substack{a \leqslant t \leqslant b \\
x, y \in \mathbb{R}}} \omega_{0}\left(h, K_{2}(t, \cdot, x, y)\right)\left\|p_{2}\right\|_{1}+ \\
+2 \rho\left(\omega_{0}\left(h, f_{1}\right)+\omega_{0}\left(h, f_{2}\right)+\omega\left(h, H_{1}\right)+\omega\left(h, H_{2}\right)\right) .
\end{aligned}
$$

Таким образом, метод согласован, если

$$
\lim _{h \rightarrow 0}\left(\max _{1 \leqslant i \leqslant n}\left\|\Delta\left(h, t_{i}\right)\right\|\right)=0 .
$$

Далее, докажем сходимость; для этого определим ошибку дискретизации следующим образом:

$$
\zeta_{i}=\left(\begin{array}{c}
\xi_{i} \\
\hat{\xi}_{i}
\end{array}\right), \quad \text { где } \quad\left\{\begin{array}{l}
\xi_{i}=X_{i}-x\left(t_{i}\right) \\
\hat{\xi}_{i}=Y_{i}-y\left(t_{i}\right)
\end{array}\right.
$$

Метод называется сходящимся, если

$$
\lim _{h \rightarrow 0}\left(\max _{0 \leqslant i \leqslant n}\left\|\zeta_{i}\right\|\right)=0 .
$$


Теорема 5. Предположим, что выполнены условия (H1), (H2) и (Н3), а интервал $[a, b]$ разбит на конечно число подынтервалов $\left[a=T_{0}, T_{1}\right],\left[T_{1}, T_{2}\right], \ldots,\left[T_{m-1}, T_{m}=b\right]$. причем $j_{v} \leqslant T_{v} / h-$ наибольшее челое число, $\mu_{i j}=\hat{\mu}_{i j}=0$ при $j>i$, а веса $\mu_{i j}, \hat{\mu}_{i j}$ удовлетворлют следующим условиям:

$$
\sum_{j=j_{v}}^{j_{v+1}-1}\left|\frac{\max \left(L_{1}, \tilde{L}_{1}\right) \mu_{i j}+\max \left(L_{2}, \tilde{L}_{2}\right) \hat{\mu}_{i j}}{1-\left[\max \left(L_{1}, \tilde{L}_{1}\right) \mu_{i i}+\max \left(L_{2}, \tilde{L}_{2}\right) \hat{\mu}_{i i}\right]}\right| \leqslant 2 \rho<1, \quad i \geqslant 1,0 \leqslant v \leqslant m .
$$

Если указанное разбиение не зависит от $h$, то

$$
\max _{1 \leqslant i \leqslant n}\left\|\zeta_{i}\right\| \leqslant\left(\frac{1}{1-2 \rho}\right)^{m} \max _{1 \leqslant i \leqslant n}\left\|\Delta\left(h, t_{i}\right)\right\| \underset{h \rightarrow 0}{\longrightarrow} 0 .
$$

Доказательство. Имеем

$$
\begin{aligned}
& \xi_{i}=\sum_{j=0}^{i} \mu_{i j}\left[K_{1}\left(t_{i}, t_{j}, X_{j}, Y_{j}\right)-K_{1}\left(t_{i}, t_{j}, x\left(t_{j}\right), y\left(t_{j}\right)\right)\right]-\delta\left(h, t_{i}\right), \\
& \hat{\xi}_{i}=\sum_{j=0}^{i} \hat{\mu}_{i j}\left[K_{2}\left(t_{i}, t_{j}, X_{j}, Y_{j}\right)-K_{2}\left(t_{i}, t_{j}, x\left(t_{j}\right), y\left(t_{j}\right)\right)\right]-\hat{\delta}\left(h, t_{i}\right) .
\end{aligned}
$$

Следовательно,

$$
\left\|\zeta_{i}\right\| \leqslant \sum_{j=0}^{i}\left[\max \left(L_{1}, \tilde{L}_{1}\right) \mu_{i j}+\max \left(L_{2}, \tilde{L}_{2}\right) \hat{\mu}_{i j}\right]\left\|\zeta_{j}\right\|+\left\|\Delta\left(h, t_{i}\right)\right\| .
$$

Для малых $h$ имеем

$$
\left\|\zeta_{i}\right\|_{1} \leqslant \sum_{j=0}^{i-1} \frac{\max \left(L_{1}, \tilde{L}_{1}\right) \mu_{i j}+\max \left(L_{2}, \tilde{L}_{2}\right) \hat{\mu}_{i j}}{1-\left[\max \left(L_{1}, \tilde{L}_{1}\right) \mu_{i i}+\max \left(L_{2}, \tilde{L}_{2}\right) \hat{\mu}_{i i}\right]}\left\|\zeta_{j}\right\|+\frac{\left\|\Delta\left(h, t_{i}\right)\right\|}{1-\left[\max \left(L_{1}, \tilde{L}_{1}\right) \mu_{i i}+\max \left(L_{2}, \tilde{L}_{2}\right) \hat{\mu}_{i i}\right]} .
$$

Для завершения доказательства остается применить [6, Theorem 8.1].

\section{4. Численные примеры.}

Пример 1. Рассмотрим следующую систему интегральных уравнений Вольтерры со слабо сингулярным ядром:

$$
\left\{\begin{array}{l}
x(t)=\int_{a}^{t}(t-s)^{-1 / 8} \frac{t^{2}\left(2 e^{-s}+1\right)}{e^{-x(s)}+e^{-y(s)}+1} d s+f_{1}(t) \\
y(t)=\int_{a}^{t}(t-s)^{-2 / 7} \frac{e^{-t}(t+s)}{\sin (x(s))^{2}+\cos (y(s))^{2}+1} d s+f_{2}(t)
\end{array}\right.
$$

здесь $t \in[0,1]$, а ядра, имеющие вид

$$
\begin{aligned}
K_{1}(t, s, x, y) & =\frac{t^{2}\left(2 e^{-s}+1\right)}{x^{2}+y^{2}+t^{2}}, & K_{2}(t, s, x, y) & =\frac{e^{-t}(t+s)}{\sin (x)^{2}+\cos (y)^{2}+1}, \\
p_{1}(t, s) & =(t-s)^{-1 / 8}, & p_{2}(t, s) & =(t-s)^{-2 / 7} .
\end{aligned}
$$

удовлетворяют условиям $(\mathrm{H} 1),(\mathrm{H} 2)$ и (Н3) при $L_{1}=\tilde{L_{1}}=M_{1}=3, L_{2}=\tilde{L_{2}}=M_{2}=2$. Если положить

получим $x(t)=t, y(t)=t$.

$$
f_{1}(t)=t-\frac{8}{7} t^{23 / 8}, \quad f_{2}(t)=t-\frac{133}{120} e^{-t} t^{12 / 7}
$$

Величины $X_{i}$ и $Y_{i}$ не поддаются точному вычислению, но могут быть найдены приближенно при помощи итерационного метода Банаха при условии остановки $\left\|X_{\text {new }}-X_{\text {old }}\right\| \leqslant 10^{-7}$. 
Таблица 1. Численные результаты для примера 1

\begin{tabular}{|r|c|}
\hline \multicolumn{1}{|c|}{$n$} & $E(h)$ \\
\hline 10 & $6,4097 \cdot 10^{-3}$ \\
50 & $6,4419 \cdot 10^{-4}$ \\
100 & $2,4559 \cdot 10^{-4}$ \\
500 & $2,6617 \cdot 10^{-5}$ \\
1000 & $1,0250 \cdot 10^{-5}$ \\
1500 & $5,8676 \cdot 10^{-6}$ \\
\hline
\end{tabular}

Таблица 2. Численные результаты для примера 2

\begin{tabular}{|r|c|}
\hline \multicolumn{1}{|c|}{$n$} & $E(h)$ \\
\hline 10 & $2,9003 \cdot 10^{-3}$ \\
50 & $2,4063 \cdot 10^{-4}$ \\
100 & $8,4262 \cdot 10^{-5}$ \\
500 & $7,4798 \cdot 10^{-6}$ \\
1000 & $2,6421 \cdot 10^{-6}$ \\
1500 & $1,4377 \cdot 10^{-6}$ \\
\hline
\end{tabular}

В таблице 1 приведены оценки ошибки, т.е. разности точного и приближенного решений:

$$
E(h)=\max _{0 \leqslant i \leqslant n}\left|X_{i}-x\left(t_{i}\right)\right|+\max _{0 \leqslant i \leqslant n}\left|Y_{i}-y\left(t_{i}\right)\right|,
$$

Отметим, что разность точного и приближенного решением стремится к нулю при $n \rightarrow \infty$; это показывает сходимость и эффективность нашего метода.

Пример 2. Рассмотрим следующую систему интегральных уравнений Вольтерры со слабо сингулярными ядрами:

$$
\left\{\begin{array}{l}
x(t)=\int_{a}^{t}(t-s)^{-1 / 2} \frac{2 s \ln (1+t)}{\sqrt{x^{2}(s)+y^{2}(s)+1}} d s+f_{1}(t), \\
y(t)=\int_{a}^{t}(t-s)^{-1 / 5}\left(t+s^{2}\right) \cos \left(\frac{1}{x^{2}(s)+y^{2}(s)+1}\right) \sin (t) d s+f_{2}(t) ;
\end{array}\right.
$$

здесь $t \in[0,1]$, а ядра, имеющие вид

$$
\begin{aligned}
K_{1}(t, s, x, y) & =\frac{2 s \ln (1+t)}{\sqrt{x^{2}+y^{2}+1}}, & K_{2}(t, s, x, y) & =\left(t+s^{2}\right) \cos \left(\frac{1}{x^{2}+y^{2}+1}\right) \sin (t), \\
p_{1}(t, s) & =(t-s)^{-1 / 2}, & p_{2}(t, s) & =(t-s)^{-1 / 5}
\end{aligned}
$$

удовлетворяют условиям (Н1), (Н2) и (Н3) при $L_{1}=\tilde{L_{1}}=2 \sqrt{2}, M_{1}=\ln 2, L_{2}=\tilde{L_{2}}=4, M_{2}=2$. Если положить

$$
f_{1}(t)=\sin (t)-\frac{4 \sqrt{2}}{3} t^{3 / 2} \ln (1+t), \quad f_{2}(t)=\cos (t)-\frac{5}{252} \cos \left(\frac{1}{2}\right) \sin (t)\left(25 t^{2}+63\right) t^{4 / 5} .
$$

получим $x(t)=\sin (t), y(t)=\cos (t)$.

Величины $X_{i}$ и $Y_{i}$ не поддаются точному вычислению, но могут быть найдены приближенно при помощи итерационного метода Банаха при условии остановки $\left\|X_{\text {new }}-X_{\text {old }}\right\| \leqslant 10^{-7}$.

В таблице 2 приведены оценки ошибки (19). На рис. 1 показаны графики точного и приближенного решений. Как и в предыдущем примере, разность точного и приближенного решением стремится к нулю при $n \rightarrow \infty$, что подтверждает сходимость и эффективность метода.

5. Заключение. В данной работе проведено обобщение результатов, полученных в [4] для нелинейного слабо сингулярного интегрального уравнения Вольтерра, на систему уравнений. Для доказательства существования и единственности решения использована теорема Шаудера о неподвижной точке. Для численного исследования применен метод интегрирования произведения, позволяющий устранить сингулярность ядер, что дает возможность аппроксимировать решение этой системы. 

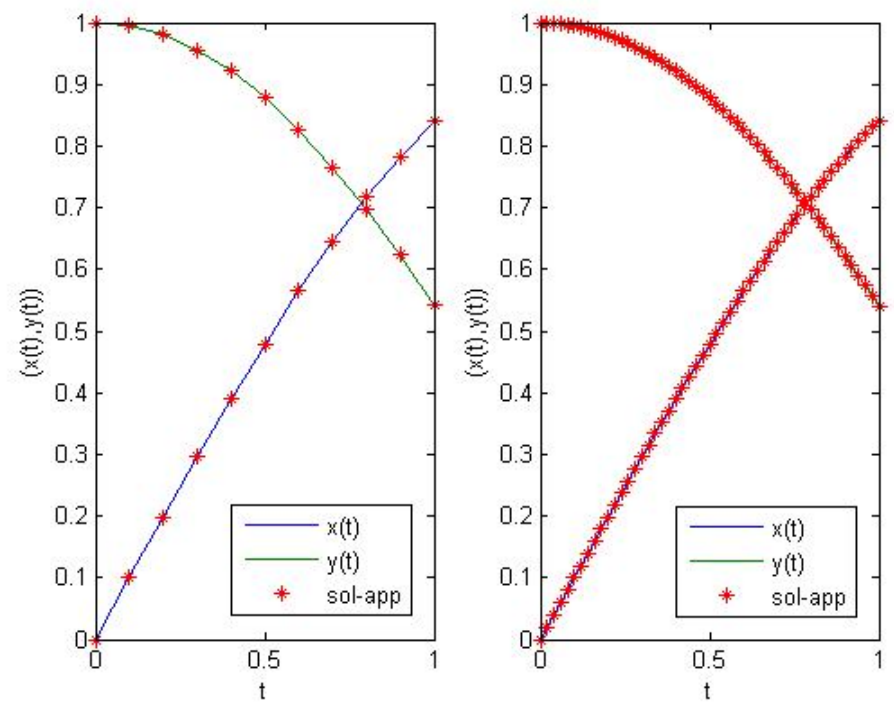

Рис. 1. Точное и приближенное решения системы (P1) при $n=10$ и $n=50$

\section{СПИСОК ЛИТЕРАТУРЫ}

1. Ahues M., Largillier A., Titaud $O$. The roles of a weak singularity and the grid uniformity in relative error bounds// Numer. Funct. Anal. Optim. — 2001. — 22, № 7. — P. 789-814.

2. Atkinson K., Han W. Theoretical Numerical Analysis. - New York: Springer-Verlag, 2009.

3. Brunner H., van der Houwen P. J. The numerical solution of Volterra equations// Math. Comput. — 1988. - 51, № 183 . - P. 379.

4. Ghiat M., Guebbai H. Analytical and numerical study for an integro-differential nonlinear equation with weakly singular kernel// Comput. Appl. Math. - 2018. — 37, № 4. — P. 4661-4674.

5. Guebbai H., Aissaoui M. Z., Debbar I., Khalla B. Analytical and numerical study for an integro-differential nonlinear Volterra equation// Appl. Math. Comput. — 2014. — 229. — P. 367-373.

6. Linz P. Analytical and Numerical Methods for Volterra Equations. - Philadelphia: SIAM, 1985.

7. Maleknejad K., Torabi P., Sauter S. Numerical solution of a nonlinear Volterra integral equation// Vietnam J. Math. — 2015. - 44, № 1. - P. 1-24.

8. Maleknejad K., Torabi P., Mollapourasl R. Fixed point method for solving nonlinear quadratic Volterra integral equations// Comp. Math. Appl. - 2011. - 62, № 6. - P. 2555-2566.

9. Segni S., Ghiat M., Guebbai H. New approximation method for Volterra nonlinear integro-differential equation// Asian-Eur. J. Math. — 2019. — 12, № 1. - 1950016.

Ghiat Mourad

Universite 8 Mai 1945, Guelma, Algeria

E-mail: mourad.ghi24@gmail.com; ghiat.mourad@univ-guelma.dz

Kamouche Soumia

Universite 8 Mai 1945 Guelma, Algeria

E-mail: soumia.kamouche@gmail.com

Khellaf Ammar

Universite 8 Mai 1945, Guelma, Algeria

E-mail: amarlasix@gmail.com; khellaf.ammar@univ-guelma.dz

Merchela Wassim

Тамбовский государственный университет им. Г. Р. Державина

E-mail: merchela.wassim@gmail.com 